\title{
The Key Role of Touch in Non-Visual Mobile Interaction
}

\author{
João Benedito, Tiago Guerreiro, Hugo Nicolau, Daniel Gonçalves \\ Technical University of Lisbon / INESC-ID \\ R. Alves Redol, 9, 1000-029, Lisbon, Portugal
}

+351214233565

jpmlb@ist.utl.pt, \{tjvg, hman\}@vimmi.inesc-id.pt, daniel.goncalves@inesc-id.pt

\begin{abstract}
Mobile devices are designed mostly to fit users with no particular disability. Tactile affordances are neglected at the expense of more attractive stylish interfaces and assistive solutions are stereotypical, also facing disabilities with a narrow perspective. A blind user is presented with screen reading software to overcome the inability to receive feedback from the device. However, these solutions go only half-way. In the absence of sight other capabilities stand up. Above all, the sense of touch plays an essential role while interacting with physical keypads. To empower these users, a deeper understanding of their capabilities and how they relate with technology is mandatory. We propose a user-product compatibility approach, taking in account that blind users have different tactile attributes. We expect to correlate the user's tactile sensitivity and keypad demands, enabling informed keypad design and selection.
\end{abstract}

\section{Categories and Subject Descriptors}

H.5.2 [Information interfaces and Presentation]: User Interfaces -input devices and strategies, user-centered design.

\section{General Terms}

Design, Experimentation, Human Factors.

\section{Keywords}

Tactile sensitivity, blind, mobile accessibility, assessment.

\section{INTRODUCTION}

Mobile phones are increasingly present in our day-to-day, making them often indispensable tools. They offer us the ability to communicate with whomever we want, whenever and wherever we are. They are responsible for a large increase in communication bandwidth and hence powerful tools of social inclusion. However, technological progress led us to more attractive and smaller devices, without in-depth understanding of their limitations and requirements for an adequate user experience. The begotten devices and interfaces present demands likely to affect their accessibility to the overall population and particularly for disabled users. A blind person faces several challenges when interacting with mobile devices. This population depends mainly on tactile sensitivity to interact with mobile phones; however, efforts have not been made to explore the influence of touch in non-visual mobile interaction.

Additionally, the tactile sensitivity in the blind population can vary widely from person to person. One of the main reasons for

Copyright is held by the author/owner(s). MobileHCI 2010 September 7 - 10, 2010, Lisbon, Portugal. ACM 978-1-60558-835-3/10/09 this is that $82 \%$ of the blind are more than 50 years old and that there is an exponential decrease in tactile sensitivity with age [1]. Furthermore, clinical states that lead to blindness, such as diabetic retinopathy, may also have other collateral damages, one of them being peripheral neuropathy, a decrease in peripheral sensitivity.

Current mobile solutions for blind users resort to the usage of screen readers, replacing the visual feedback by its auditory representation. However, the feedback is restricted to the information presented onscreen. Thus, besides the effort to memorize the keypad layout, interaction is dependent on keypad recognition abilities, giving the sense of touch the upmost relevance in the absence of visual feedback. Still, there is no understanding of which is the best device for a particular user and his touch capabilities, thus restricting the user experience and an informed design of more inclusive mobile keypads.

Our goal is to bridge this gap and relate the users' tactile sensitivity with keypad characteristics and their demands. In this paper, we present our approach as well as a tactile sensitivity preliminary assessment showing that this attribute is variable and far from perfect (against popular belief) between blind users.

\section{THE RELEVANCE OF TOUCH}

The capability-demand theory builds on the concepts of user capability and product demand and aims to analyze user-product compatibility, i.e. an assessment and comparison of the sensory, cognitive and motor demand made by a product in relation to the ability levels of the expected user population [2]. We will focus on a particular capability, touch, and the demands it relates to.

Regarding user capabilities, tactile sensitivity will be evaluated in term of spatial acuity and pressure sensitivity. As to devices, we categorize mobile keypads in terms of key size, spacing, height, material and labels. With both ends quantified, we will be able to assert relations between them and aim at a match between user capabilities and product demands. This way, we will provide both the tools for mobile designers, showing which designs are most effective/inclusive, and for blind users, identifying the most appropriate keypads for each one.

\subsection{Tactile Sensitivity}

There are three key components for assessing low-level tactile sensitivity in a person's fingers. These components are the pressure sensitivity, spatial acuity and thickness discrimination [3]. We will focus on pressure sensitivity and spatial acuity as we consider those to be the involved characteristics in mobile keypad interaction. Further, we include a functional assessment consisting on the identification of the number of dots of random Braille characters printed on a piece of paper. Tactile sensitivity will be measured in the thumb, index and middle fingers, the ones the target users employ to interact with the keypad. 


\subsection{Mobile device demands}

Each mobile phone will be categorized according to relevance and confirmed by questionnaires performed to the users on the likes and dislikes considering their current and past mobile phones. Table 1 summarizes the characteristics relevancy.

Table 1: Characteristics chosen to identify product demands

\begin{tabular}{|c|c|}
\hline Characteristic & Justification \\
\hline Key size & $\begin{array}{c}\text { Keys too small are harder to identify, } \\
\text { especially if the spacing between them is low }\end{array}$ \\
\hline Key spacing & $\begin{array}{c}\text { When the spacing is too small it is harder to } \\
\text { identify different keys }\end{array}$ \\
\hline Key height & $\begin{array}{c}\text { It is hard to distinguish a key with a low } \\
\text { height from the base of the cell phone }\end{array}$ \\
\hline Material & $\begin{array}{c}\text { If the key's material and device base are } \\
\text { contrasting it is easier to tell them apart }\end{array}$ \\
\hline Label & $\begin{array}{c}\text { The label must have sufficient relief and/or in } \\
\text { sufficient size/quantity to be identified. }\end{array}$ \\
\hline
\end{tabular}

Considering the capability-demand theory, for a successful user experience, product demands must be smaller than user capabilities. It is thus required to identify mobile phone keypad's demands regarding tactile sensitivity. To find those relations, besides assessing the users' tactile levels we will evaluate their low-level effectiveness and efficiency with diverse mobile devices. We will measure the time between the stimuli and key presses, and all interactions will be recorded to get the type and number of errors. Then, it will be possible to find relations between tactile sensitivity and keypads' physical characteristics.

\subsection{Predicting user-device match}

We will confirm if in fact the users have different tactile levels, if they affect interaction, which assessments tell us the most, which keypad features have the greatest impact and how they are related. With this we will be in position to provide a user-product predictive model. The goal is to predict, for each user, which mobile keypad is most suited for his capabilities. Further, we will be able to predict which devices are more inclusive, considering a wide user sample. This model will be evaluated with marketavailable devices. Our solution provides many advantages. For instance, a design company can use it in early design stages to make decisions, and therefore avoid finding, late in the process, the accessibility challenges it encloses. Moreover, it enables assessing a device's inclusiveness without empiric evaluations.

\section{PRELIMINARY STUDIES}

Up until now, we have focused our efforts in evaluating the user dimension. This has been done by measuring the tactile sensitivity of 18 blind people. Pressure sensitivity was measured resorting to Semmes-Weinstein mono-filaments [3]. In this method, if the users can feel the touch of the monofilament when it is folded against their skin, the pressure sensibility is equal to the resistance of the monofilament. Spatial acuity was measured using a DiskCriminator [4]. This is an orthogonal device with a pair of filaments spaced by distances going from 2 to $15 \mathrm{~mm}$ on each side. The user is asked to identify, starting on the smallest distance, if he is touched by one or two points. The spatial acuity is equal to the minimum distance where the user identifies at least 7 out of 10 single or double touches. Figure 1 presents the results showing different tactile sensitivities between users and even between each user's fingers.
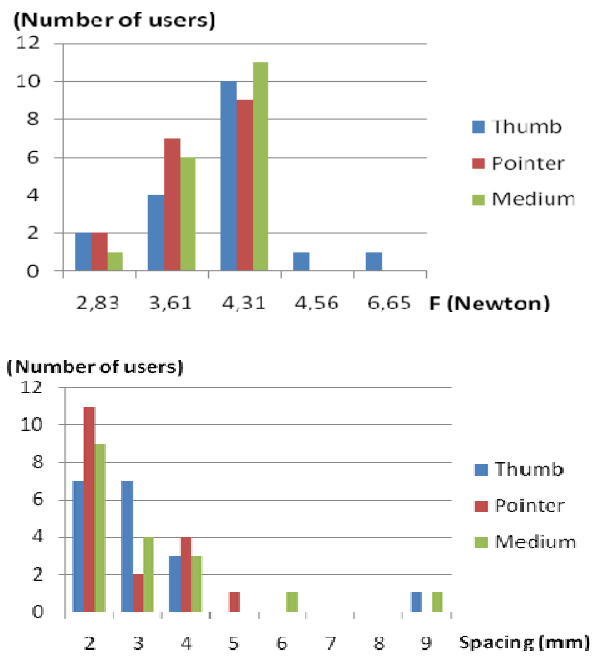

Figure 1 - Pressure Sensitivity (Up) and Spatial Acuity (Down)

The next step is to categorize diverse phones and evaluate user performance while interacting with them. Then, by evaluating the results, we will be able to identify the relationships between the levels of tactile sensitivity and mobile phone characteristics, and provide a predictive model enclosing the achieved knowledge. We will then be able to bridge a growing gap, where blind users are leaded to use devices unsuitable for their individual differences.

\section{CONCLUSION}

The sense of touch is of extreme relevance for a blind user. Even mobile solutions for the blind overlook this evidence. We focus on tactile sensitivity and how different tactile levels reflect in different mobile interaction abilities. We aim to understand how tactile aptitudes relate to keypad attributes, and provide a predictive model to improve device design and selection.

\section{ACKNOWLEDGMENTS}

This work was supported by FCT through the PIDDAC Program funds. Hugo Nicolau and Tiago Guerreiro were supported by FCT, grants SFRH/BD/46748/2008 and SFRH/BD/28110/2006.

\section{REFERENCES}

[1] WHO (2007). Magnitude and causes of visual impairment.

[2] Persad, U., et al. (2007). Characterizing user capabilities to support inclusive design evaluation. Universal Access in the Information Society, 6(2):119-135.

[3] Tremblay, F., et al. (2005). Relationships between age, tactile sensibility and magnitude of contact forces. Experimental Brain Research:155-164.

[4] Mackinnon S, Dellon A (1985). Two-point discrimination tester. Journal of Hand Surgery. 10A:906-7 